\title{
Prognostic Value of Twist-1, E-cadherin and EZH2 in Prostate Cancer: An Immunohistochemical Study
}

\author{
Aziza E. ABDELRAHMAN', Shimaa A. ARAFA', Ragab A. AHMED² \\ Department of 'Pathology and 'Urology, Zagazig University, Faculty of Medicine, ZAGAZIG, EGYPT
}

\begin{abstract}
Objective: There is an urgent need to identify molecular biomarkers rather than clinical markers to distinguish aggressive prostate cancer from the indolent majority for proper treatment and accurate prediction of prognosis. We aimed to investigate the immunohistochemical expression of epithelial-mesenchymal transition (EMT)-related molecules (Twist-1 and E-cadherin) and the stem cell marker EZH2 in prostate cancer and to assess their ability to identify the high-risk patients, in a trial to explore their prognostic implications.

Material and Method: Immunohistochemical expression of Twist-1, E-cadherin and EZH2 in 50 specimens of prostate cancer and 20 cases of benign prostatic hyperplasia were studied. The relationship between their expression and the clinicopathological variables, biochemical recurrence, and biochemical progression-free survival were investigated.

Results: Our results revealed that high Twist-1, as well as high EZH2 expression, was strongly associated with higher pre-treatment PSA level, Gleason score $\geq 7$, advanced tumor stage, lymph node involvement, distant metastasis and biochemical progression. Aberrant E-cadherin expression was significantly associated with higher pre-treatment PSA level, Gleason score $\geq 7$, advanced tumor stage, lymph node involvement, and distant metastasis. A significant positive correlation between Twist- 1 and EZH2 expression was found ( $\mathrm{p}<0.001)$, while E-cadherin expression showed a negative correlation with both markers $(\mathrm{p}<0.001)$. A significant association was found between high Twist- 1 , high EZH2\& aberrant E-cadherin expression, and shorter biochemical progression-free survival.
\end{abstract}

Conclusion: The high Twist-1 expression, aberrant E-cadherin and high EZH2 expression in primary prostate cancer are considered as adverse prognostic markers of an aggressive tumor with high metastatic potential. Assessment of their expression level would contribute to the accurate prediction of biochemical progression.

Key Words: EZH2, Twist-1, E-cadherin, Prostate cancer, Epithelial-mesenchymal transition.

\section{INTRODUCTION}

Prostate cancer (PC) is the most common neoplasm of males and despite the progressive decline in its incidence and mortality, it is still the second common cause of cancerrelated death among men (1). The National Comprehensive Cancer Network (NCCN) guidelines defined the high-risk localized prostate cancer as initial PSA $>20 \mathrm{ng} / \mathrm{ml}$, clinical stage $\geq \mathrm{T} 3 \mathrm{a}$ and Gleason score $\geq 8$ (2). However, these clinical markers do not adequately discriminate between indolent tumors and those that will progress to be metastatic, so efforts are now directed towards using a combination of biological rather than clinical markers to predict prognosis and response to therapy (3).

Several studies have reported that epithelial-mesenchymal transition (EMT) and cancer stem cells (CSCs) play a key role in metastasis and drug resistance of prostatic carcinoma. Understanding the determinants responsible for EMT and CSCs will be essential to develop new promising therapies for PC in the future (4).

(Turk Patoloji Derg 2017, 33:198-210)

Received : 25.12.2016 Accepted : 08.02.2017
Epithelial-mesenchymal transition is a process found in embryogenesis and tumor invasion which is thought to be a general character of cancer stem cell and progenitor cell populations. They lose their polarity and intercellular adhesion molecules to transform into invasive and migrating mesenchymal cells (5).

Castration can induce EMT that may enhance the stemness of CSCs to facilitate aggressive and metastatic behavior, which in turn results in castration-resistance and metastasis (6). Recent studies have demonstrated that EMT plays a critical role in tumor recurrence and that it is tightly linked to the biology of cancer stem cells or cancer-initiating cells (7).

The major changes of epithelial-mesenchymal transition occur at the molecular level before tumor morphology and the utilization of these early molecular changes may be crucial in predicting the prognosis of cancer (8). The process of EMT includes upregulation of pro-EMT proteins such as Twist-1, N-cadherin and fibronectin, as well as

Correspondence: Aziza E. ABDELRAHMAN

Department of Pathology, Zagazig University, Faculty of Medicine,

ZAGAZIG, EGYPT

E-mail: azaelsayed@gmail.com Phone: +00 201068743218 
down-regulation of EMT depressors as desmoplakin, cytokeratins, and E-cadherin (9).

Twist-1, a member of the basic helix-loop-helix transcription factors, was reported as a key factor in EMT promoting metastasis of a cancer cell. Previous studies have been reported that Twist-1 is a master regulator of embryonic morphogenesis that controls cell migration and differentiation under various physiological conditions and promotes EMT under some pathological conditions including cancer (10).

The Twist-1 biomarker plays a crucial role in tumor growth, cell invasion, and metastasis through regulation of cancerrelated functions, such as angiogenesis, and degradation of the extracellular matrix in various malignancies. In addition, it promotes EMT by repressing the expression of E-cadherin leading to disassembly of adherens junctions and increased migratory potential (11).

E-cadherin is a $\mathrm{Ca}^{2}$-dependent transmembrane protein that mediates cellular adhesion in normal epithelium via interactions with $\beta$-catenin in the cytoplasm. Its expression level is negatively correlated with the development of EMT and tumor invasion. Loss or aberrant expression of E-cadherin is related to PC progression, metastasis, and poor prognosis through two different mechanisms, cell-cell adhesion and paracrine action (12). Cleavage of E-cadherin ectodomain has been shown to create an sE-cad fragment, capable of inducing EMT, invasion, and proliferation in a paracrine manner via EGFR signaling (13).

Epithelial-mesenchymal transition could potentially offer a satisfactory explanation for the origin of CSCs, but the molecular mechanisms linking EMT to stemness are still unclear. The cancer stem cell theory contributes to a second explanation for the relapse and resistance that occurs in multiple tumors after therapy (7).

Enhancer of zeste homolog-2 (EZH2), a catalytic subunit of Polycomb Repressive Complex 2 (PRC2) responsible for histone H3-lysine 27 methylation, regulates gene transcription and chromatin structure. Some studies suggest that EZH2 plays a crucial role in stem cell renewal, maintenance, and differentiation into specific cell lineages (14). Moreover, it is overexpressed in aggressive solid tumors, including prostate cancer, and may be a useful prognostic marker in clinical practice with chemotherapeutic agents that specifically target the enzyme (15). Some studies have also suggested that its oncogenic activity is thought to be mainly mediated by silencing tumor suppressor genes.
EZH2 is implicated in EMT activation by the inhibition of E-cadherin expression, and its upregulation represents one of the most frequent epigenetic alterations during prostate cancer progression (16).

Development of drugs either inhibiting EMT, CSCs or enhancing the expression of epithelial markers could be a novel strategy for PC therapy in the future (4). In the present study, we aimed to investigate the immunohistochemical expression of EMT-related molecules (Twist-1 and E-cadherin) and the stem cell marker EZH2 in PC and to assess their ability to identify high-risk patients, in a trial to explore their prognostic implications.

\section{MATERIALS and METHODS}

\section{Tissue Specimens}

In this retrospective cohort study, fifty formalin-fixedparaffin-embedded tissue specimens of PC and twenty cases of benign prostatic hyperplasia (BPH) as control cases were collected from the archive of Pathology Department, Zagazig University Hospitals, Egypt. The tissue specimens were obtained either by transrectal ultrasound-guided prostatic needle biopsy ( 15 cases, 6 paraffin blocks for each), open prostatectomy (5 cases), or radical prostatectomy (30 cases) at the Urology Department during the period from November 2013 to December 2015. The clinicopathological data were retrieved from patients' files including age; initial prostate-specific antigen (PSA) level, tumor stage (pT), Gleason score, nodal metastasis, distant metastasis and follow-up data. Distant metastasis was established by clinical features, radiology, and radio-isotopic bone scan. Metastasis to lymph nodes was diagnosed by histological examination beside PSA staining of the resected lymph nodes. None of the patients had received preoperative androgen ablation before surgery. Histopathologic diagnosis of all cases was reviewed by two pathologists (AE, SA) to unify the reproducibility of the diagnosis. According to World Health Organization classification of prostate cancer and the revised Gleason grading system, PCa specimens were divided into low-grade $(<7)$ and high-grade cancers $(\geq 7)$ (17) Tumor stages $(\mathrm{pT})$ were defined based on the TNM prostate cancer staging system (18). The mean follow-up period of cancer patients was $23.32 \pm 9.18$ months (range 3-36 m). All patients were followed up with a digital rectal examination, imaging studies and serum PSA assay every 3 months in the first year, semi-annually thereafter Biochemical progression was defined as persistent or rising PSA level of $>0.2 \mathrm{ng} / \mathrm{ml}$ in two consecutive blood samples over a 2-month period. 


\section{Immunohistochemical Procedure}

Paraffin sections of 3-5 $\mu \mathrm{m}$ were stained using the streptavidin-biotin-peroxidase technique. The tissue sections were deparaffinized in xylene and rehydrated through graded alcohol. Epitope retrieval by boiling in citrate buffer ( $\mathrm{pH}$ 6.0) for $20 \mathrm{~min}$ was done and then washed in phosphate buffer saline (PBS). Endogenous peroxidase activity was blocked by incubation of slides in $3 \%$ hydrogen peroxide for 20 minutes. After washing with PBS, blocking serum was applied for $10 \mathrm{~min}$. At room temperature, the tissue sections were incubated overnight with an anti-EZH2 antibody (1:100, BD Biosciences, CA); Rabbit polyclonal anti-Twist antibody (1:50, ab50581; Abcam, Cambridge, $\mathrm{UK})$ and monoclonal anti-E-cadherin antibody (1:50; Dako, Carpinteria, CA, UK). After rinsing in PBS, the tissues were incubated with a biotin-conjugated secondary antibody (Lab Vision Corporation, Fermont, USA) and then incubated using the streptavidin-biotin system for 1 hour at room temperature. The sections were incubated with diaminobenzidine $(\mathrm{DAB})$ for 15 minutes then rinsed with distilled water. Finally, the slides were counterstained with Meyer's hematoxylin, dehydrated and mounted. Negative controls were made by substitution of primary antibodies with a non-immune serum. Normal prostatic glands, thyroid cancer, and breast ductal carcinoma were used as positive controls for E-cadherin, Twist-1, and EZH2, respectively.

\section{Scoring Criteria}

At the start of this study we excluded prostate cancer cases showing marked heterogeneous appearance. In addition, during the assessment of immunohistochemical expression of the markers we evaluated four representative tumor regions (high Gleason, low Gleason, central and margin) by both extension and intensity.

\section{Evaluation of Twist-1 Immunostaining}

The cytoplasmic staining intensity was scored as follows: 0 , negative; 1 , weak; 2 , medium; and 3 , strong. The staining extent was scored as follows: $0,0 \% ; 1,1-25 \% ; 2,26-50 \%$; $3,51-75 \%$; and $4,76-100 \%$. The final scores $(0-7)$ were calculated as the sum of the intensity score and extent score. The staining scores of more than 3 were considered as highexpression (19).

\section{Evaluation of E-Cadherin Immunostaining}

Membranous E-cadherin immunoreactivity was interpreted as normally or aberrantly immunoreactive. Immunoreactivity was classified as normal if the specimens showed strong or moderate membranous staining and weak or negative cytoplasmic staining in $>70 \%$ of the cells. Patterns other than those were regarded as aberrant immunoreactivity (20).

\section{Evaluation of EZH2 Immunostaining}

The nuclear staining for EZH2 in ten high power fields was recorded and calculated for each case and the percentage of positive cells was scored as follows: $0-33 \%$, score $1 ; 34$ $66 \%$, score 2 and $>67 \%$, score 3 . The staining intensity ( 0 : negative; 1: mild; 2: moderate; 3: strong) was recorded. Finally, the staining index (SI) was obtained by multiplying the two scores: SI (0-9). A final score of $\geq 4$ was considered to be high (21).

\section{Statistical Analysis}

Continuous variables were expressed as the mean \pm SD \& median (range), and categorical variables were expressed as a number (percentage). Continuous variables were checked for normality by Shapiro-Wilk test. Mann-Whitney U-test was used to compare between two groups of non-normally distributed variables. Percent of categorical variables were compared using Pearson's Chi-square test or Fisher's exact test when appropriate. The trend of change in the distribution of relative frequencies of ordinal data was compared using the chi-square test for trend. Biochemical progression-free survival (BPFS) was calculated as the time from the start of treatment to date of biochemical progression or the most recent follow-up contact that a patient was known as biochemical progression free. Stratification of BPFS was done according to markers. These time-to-event distributions were estimated using the Kaplan-Meier plot method, and compared using the two-sided exact log-rank test. All tests were two-sided. A p-value $<0.05$ was considered significant. Statistical analysis was done using SPSS 22.0 for Windows (SPSS Inc., Chicago, IL, USA) and MedCalc windows (MedCalc Software bvba 13, Ostend, Belgium). The study was approved by our local research ethics committee.

\section{RESULTS}

\section{Clinicopathological Features}

The mean age of cancer patients at the diagnosis was $62.94 \pm 8.76$ years, while the mean age of $\mathrm{BPH}$ cases was at $58.40 \pm 11.98$ years. No significant difference between the two groups concerning age was present $(p=0.138)$. Gleason score $\geq 7$ was the most common score in this cohort (56\%). Forty percent of PC patients had pT2 disease while $60 \%$ had pT3-pT4. Distant metastasis was detected in 12 cases (24\%) and lymph node involvement was observed in 24 cases (48\%). During the follow-up period, biochemical progression was noted in $30 \%$ of the patients. Mean serum 
PSA level in cancer patients was $19.8 \pm 14.02 \mathrm{ng} / \mathrm{ml}$ (range 4-50) and $2.03 \pm 1.33 \mathrm{ng} / \mathrm{ml}$ (range 0.4-5) in BPH. Other clinicopathological features of $\mathrm{PC}$ and $\mathrm{BPH}$ cases are presented in Tables I, II.

\section{Twist-1 Expression}

The majority of PC cases (54\%) showed high Twist-1 expression in the tumor cells while $100 \%$ of $\mathrm{BPH}$ specimens showed low expression $(p<0.001)$. Further investigation of the correlation between Twist-1 immunoexpression and clinicopathological features revealed that the increased expression was associated with an initial PSA level $(p<0.001)$. In addition, Twist-1 immunoreactivity was up-regulated with increased tumor grade with statistically significant difference $(p<0.001)$ (Figure 1A-C). Furthermore, a significant difference in Twist-1 expression was observed regarding the pathological tumor stage $(p=<0.001)$, LN involvement $(p<0.001)$, and distant metastasis $(p=0.003)$.

\section{E-Cadherin Expression}

All BPH specimens showed moderate to strong membranous immunoreactivity of E-cadherin (preserved pattern) (Figure 2), while 62\% cases of PC specimens had aberrant E-cadherin immunoreactivity with a significant difference between the two groups $(p<0.001)$.

Low-grade PC of Gleason score $<7$ showed aberrant E-cadherin immunoreactivity in only $27.3 \%$ of cases, while the majority of high-grade PC (89.3\%) had aberrant expression $(p<0.001)$ (Figure 3A-D). A significant correlation was found between E-cadherin expression patterns and initial PSA level $(p<0.001)$. Aberrant E-cadherin expression was more common in advanced pathological stages than the early stages $(p<0.001)$. Furthermore, loss of membranous E-cadherin expression was found in all cases associated with LN involvement and distant metastasis in contrast to those cases with negative LN and without distant metastasis $(p<0.001, p=0.002$, respectively).

\section{EZH2 Expression}

Analysis of both staining intensity and extension of nuclear EZH2 revealed that all cases of $\mathrm{BPH}$ showed a low expression level limited to the prostate epithelium and absent in the surrounding stroma, while $66 \%$ of PC cases exhibited a high

Table I: Clinicopathological features of 50 patients with prostatic carcinoma

\begin{tabular}{|c|c|c|c|c|c|}
\hline Characteristics & No. & $\%$ & Characteristics & No. & $\%$ \\
\hline Age (year) & & & $\bar{M}$ & & \\
\hline Mean \pm SD & $62.94 \pm 8.76$ & & M0 & 38 & $76 \%$ \\
\hline Median (Range) & $60.50(50-86)$ & & M1 & 12 & $24 \%$ \\
\hline$<65$ years & 31 & $62 \%$ & Twist-1 & & \\
\hline$\geq 65$ years & 19 & $38 \%$ & Low & 23 & $46 \%$ \\
\hline PSA (ng/mL) & & & High & 27 & $54 \%$ \\
\hline Mean \pm SD & $19.86 \pm 14.02$ & & E-cadherin & & \\
\hline Median (Range) & $14.50(4-50)$ & & Normal & 19 & $38 \%$ \\
\hline$<10 \mathrm{ng} / \mathrm{mL}$ & 16 & $32 \%$ & Aberrant & 31 & $62 \%$ \\
\hline$\geq 10 \mathrm{ng} / \mathrm{mL}$ & 34 & $68 \%$ & EZH2 & & \\
\hline Gleason score & & & Low & 17 & $34 \%$ \\
\hline$<7$ & 22 & $44 \%$ & High & 33 & $66 \%$ \\
\hline$\geq 7$ & 28 & $56 \%$ & Follow-up (months) & & \\
\hline $\mathrm{pT}$ & & & Mean \pm SD & $23.32 \pm 9.18$ & \\
\hline pT2 & 20 & $40 \%$ & Median (Range) & $24.50(3-36)$ & \\
\hline pT3 & 20 & $40 \%$ & Progression & & \\
\hline pT4 & 10 & $20 \%$ & Absent & 35 & $70 \%$ \\
\hline $\mathrm{pN}$ & & & Present & 15 & $30 \%$ \\
\hline $\mathrm{pN} 0$ & 21 & $42 \%$ & & & \\
\hline $\mathrm{pN} 1$ & 24 & $48 \%$ & & & \\
\hline $\mathrm{pNx}$ & 5 & $10 \%$ & & & \\
\hline
\end{tabular}

Continuous variables were expressed as mean \pm SD \& median (range). Categorical variables were expressed as number (percentage).

Progression: Biochemical progression. 

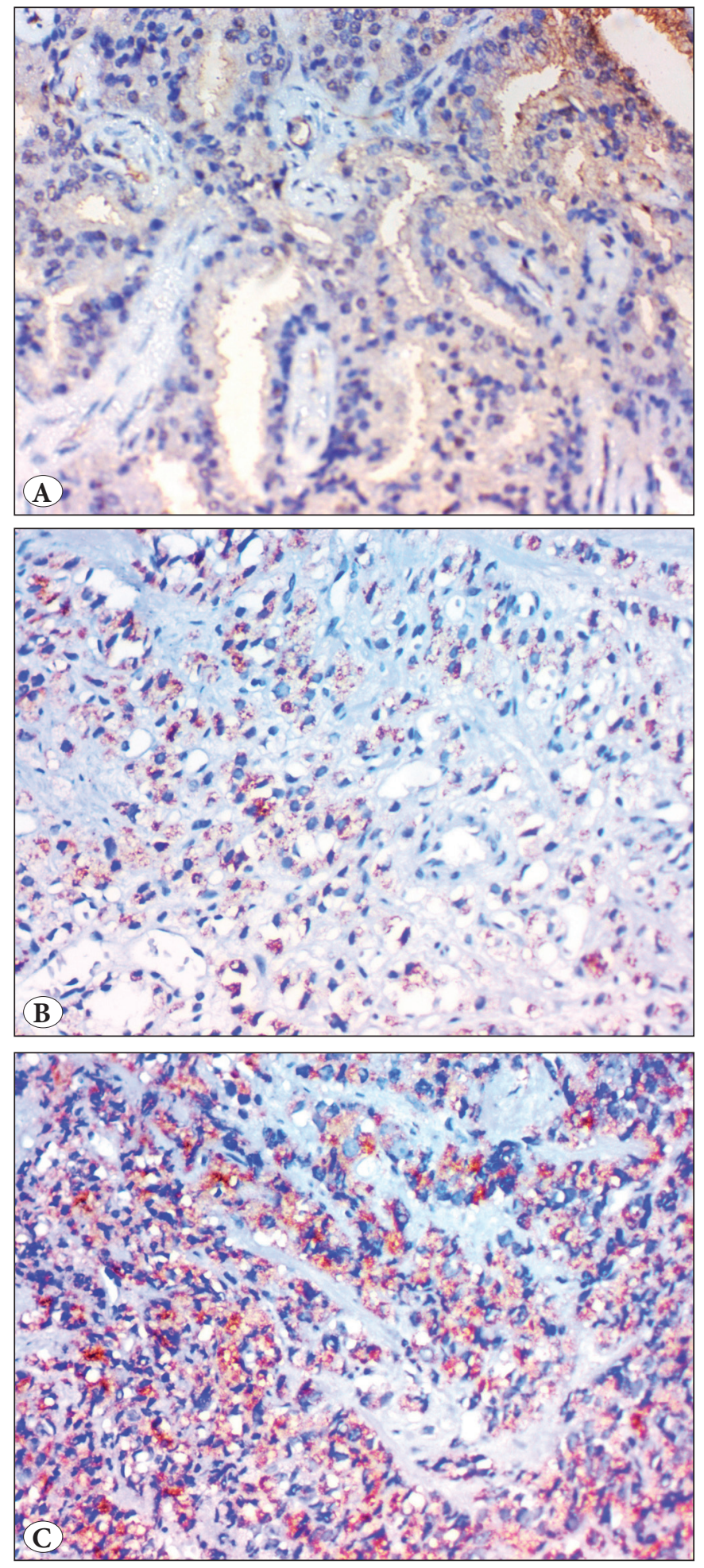

Figure 1: A) Low-grade prostate cancer (Gleason score $<7$ ) shows negative immunoreactivity of Twist-1 (IHC; x400).

B) High-grade prostate cancer (Gleason score $\geq 7$ ) shows low cytoplasmic immunoreactivity of Twist-1 (IHC; x400).

C) High-grade prostate cancer (Gleason score $\geq 7$ ) shows high cytoplasmic immunoreactivity of Twist-1 (IHC; x400). expression level in tumor cells with a significant difference between the two groups $(p<0.001)$.

Analysis of the correlation between nuclear EZH2 and clinicopathological parameters revealed that patients with high EZH2 expression had a higher initial PSA level and Gleason scores $(p<0.001, \mathrm{p}=0.001$, respectively) at presentation (Figure $4 \mathrm{~A}, \mathrm{~B})$. Moreover, it was found that all cases of advanced tumor stage (pT3, pT4), LN involvement and distant metastasis revealed high nuclear EZH2 expression.

The correlation between Twist-1, E-cadherin and EZH2 expression and the clinicopathological parameters are demonstrated in Table III.

\section{Association Between Twist-1, E-Cadherin and EZH2 Expression}

The correlation analysis of our marker expression among PC cases revealed a significant positive correlation between Twist-1 and EZH2 expression $(p<0.001)$, while E-cadherin expression showed a negative correlation with both markers $(p<0.001)$ (Table III).

\section{Association of Twist-1, E-Cadherin and EZH2 Expression with Biochemical Progression and BPFS}

During the follow-up period, 15 out of 50 patients (30\%) showed biochemical progression at the mean time of 29.4 months with 3 year BPFS in $63.7 \%$ of cases. We found that $51.9 \%$ of high Twist-1 expression, $38.7 \%$ of aberrant E-cadherin, and $42.4 \%$ of high EZH2 cases had a biochemical progression within 3 years. However, only



Figure 2: Benign prostatic hyperplasia shows strong and complete membranous immunoreactivity of E-cadherin (preserved expression), (IHC; $\mathrm{x} 400)$. 
$4.3 \%$ of low Twist- 1 expression ( $p<0.001$ ), $15.8 \%$ of normal E-cadherin expression $(p=0.086)$, and $5.9 \%$ of low EZH2 expression cases $(p=0.008)$ had a progressive disease (Table IV). Kaplan-Meier survival curve analysis revealed a significant association between high Twist-1 $(p<0.001)$, aberrant E-cadherin $(p=0.013)$ and high EZH2 expressions $(p=0.002)$ and shorter BPFS (Figure 5A-C).

\section{DISCUSSION}

Prostate cancer is the second leading cause of cancerrelated mortality in males. The transition of a subset of tumors from indolent to invasive disease is associated with a poor clinical outcome. Activation of EMT genetic program is a major risk factor for cancer progression (16). It is important to accurately predict the prognosis of cancer patients following surgical resection of the tumors using an accepted consensus to define postoperative follow-up schedule as well as additional treatment strategies (22). Therefore, individual assessment of a tumor's aggressive potential is crucial for clinical decision making. The critical question is how to identify patients with a high-risk of recurrence or progression (23).
In this study, we evaluated the expression level of Twist-1, E-cadherin and EZH2 in PC in a trial to assess their role in the pathogenesis, progression, and identification of cancer patients who will likely progress to aggressive disease and therefore need radical treatment or earlier intervention.

In the present work, Twist-1 up-regulation was observed only in PC as compared to $\mathrm{BPH}$; denoting its role in neoplastic transformation and progression of cancer. Increased expression of Twist-1 was associated with the initial PSA level and Gleason grade supporting the previous reports about its negative impact on cancer cell differentiation and histological progression (24). However, Whiteland et al. reported that Twist-1 expression was indifferent between PC and BPH. In addition, they reported indifferent Twist-1 expression between different Gleason scores. They explained this discrepancy of results by different patient demographics (25).

Furthermore, a significant positive association between Twist- 1 expression and TNM staging including $\mathrm{pT}$ stage, LN involvement, and distant metastases was demonstrated where most of the advanced stages had high Twist-1 expression. Our data agree with previous studies that found

Table II: Comparison between prostatic carcinoma and BPH as regard clinicopathological features, immunohistochemical markers.

\begin{tabular}{|c|c|c|c|c|c|}
\hline \multirow{2}{*}{ Parameters } & \multicolumn{2}{|c|}{ Prostatic carcinoma $(\mathrm{N}=50)$} & \multicolumn{2}{|c|}{ BPH $(\mathrm{N}=20)$} & \multirow{2}{*}{ p-value } \\
\hline & No. & $(\%)$ & No. & $(\%)$ & \\
\hline \multicolumn{6}{|l|}{ Age (year) } \\
\hline Mean \pm SD & $62.94 \pm 8.76$ & & $58.40 \pm 11.98$ & & \multirow{2}{*}{$0.138 \ddot{Y}$} \\
\hline Median (Range) & $60.50(50-86)$ & & $58(40-80)$ & & \\
\hline$<65$ years & 31 & $(62 \%)$ & 13 & $(65 \%)$ & \multirow{2}{*}{$0.814 \ddagger$} \\
\hline$\geq 65$ years & 19 & $(38 \%)$ & 7 & $(35 \%)$ & \\
\hline \multicolumn{6}{|l|}{ PSA (ng/mL) } \\
\hline Mean \pm SD & $19.86 \pm 14.02$ & & $2.03 \pm 1.33$ & & \multirow{2}{*}{$<0.001 \ddot{Y}$} \\
\hline Median (Range) & $14.50(4-50)$ & & $1.75(0.40-5)$ & & \\
\hline$<10 \mathrm{ng} / \mathrm{mL}$ & 16 & $(32 \%)$ & 20 & $(100 \%)$ & \multirow{2}{*}{$<0.001 \ddagger$} \\
\hline$\geq 10 \mathrm{ng} / \mathrm{mL}$ & 34 & $(68 \%)$ & 0 & $(0 \%)$ & \\
\hline \multicolumn{6}{|l|}{ Twist-1 } \\
\hline Low & 23 & $(46 \%)$ & 20 & $(100 \%)$ & \multirow{2}{*}{$<0.001 \ddagger$} \\
\hline High & 27 & $(54 \%)$ & 0 & $(0 \%)$ & \\
\hline \multicolumn{6}{|l|}{ E-cadherin } \\
\hline Normal & 19 & $(38 \%)$ & 20 & $(100 \%)$ & \multirow{2}{*}{$<0.001 \neq$} \\
\hline Aberrant & 31 & $(62 \%)$ & 0 & $(0 \%)$ & \\
\hline \multicolumn{6}{|l|}{ EZH2 } \\
\hline Low & 17 & $(34 \%)$ & 20 & $(100 \%)$ & \multirow{2}{*}{$<0.001 \neq$} \\
\hline High & 33 & $(66 \%)$ & 0 & $(0 \%)$ & \\
\hline
\end{tabular}

Continuous variables were expressed as mean \pm SD \& median (range); categorical variables were expressed as number (percentage); $\ddot{Y}$ Mann-Whitney U test; $¥$ Chi-square test; $\mathrm{p}<0.05$ is significant. 
high Twist-1 expression in PC and its correlation with disease aggressiveness and metastasis $(20,22)$.

The explanation for the significant association between Twist-1 with advanced tumor stage and the metastatic potential of the primary cancer is the transcriptional repression of E-cadherin, resulting in a loss of cellular adhesion and an enhancement of PC cell motility (26). These findings were confirmed by our results, where the highest Twist-1 expression was significantly associated with aberrant E-cadherin expression. In a previous in vitro study, Kwok et al. reported that by reducing the expression of Twist-1 in PC cell lines, E-cadherin was redistributed from the cytoplasm to the cell membrane (27). Consideration of all these data, we can guess that Twist- 1 overexpression in the PC may enhance EMT by promoting E-cadherin switching.

Kwok et al. observed that down-regulation of Twist-1 in androgen-independent PC cells increased their sensitivity to anticancer therapy and decreased their invasion and migration abilities, suggesting Twist-1 inactivation as a potential strategy to control the growth and metastasis of these cells (27). Previous studies have reported that the highest expression of Twist-1 was associated with high grade, invasion, metastases, and therefore unfavorable prognosis in different cancers as thyroid (28), and lung carcinomas (29). These results regarding Twist-1 may help to find a new therapeutic target to inhibit the invasion, progression, and metastases in PC.
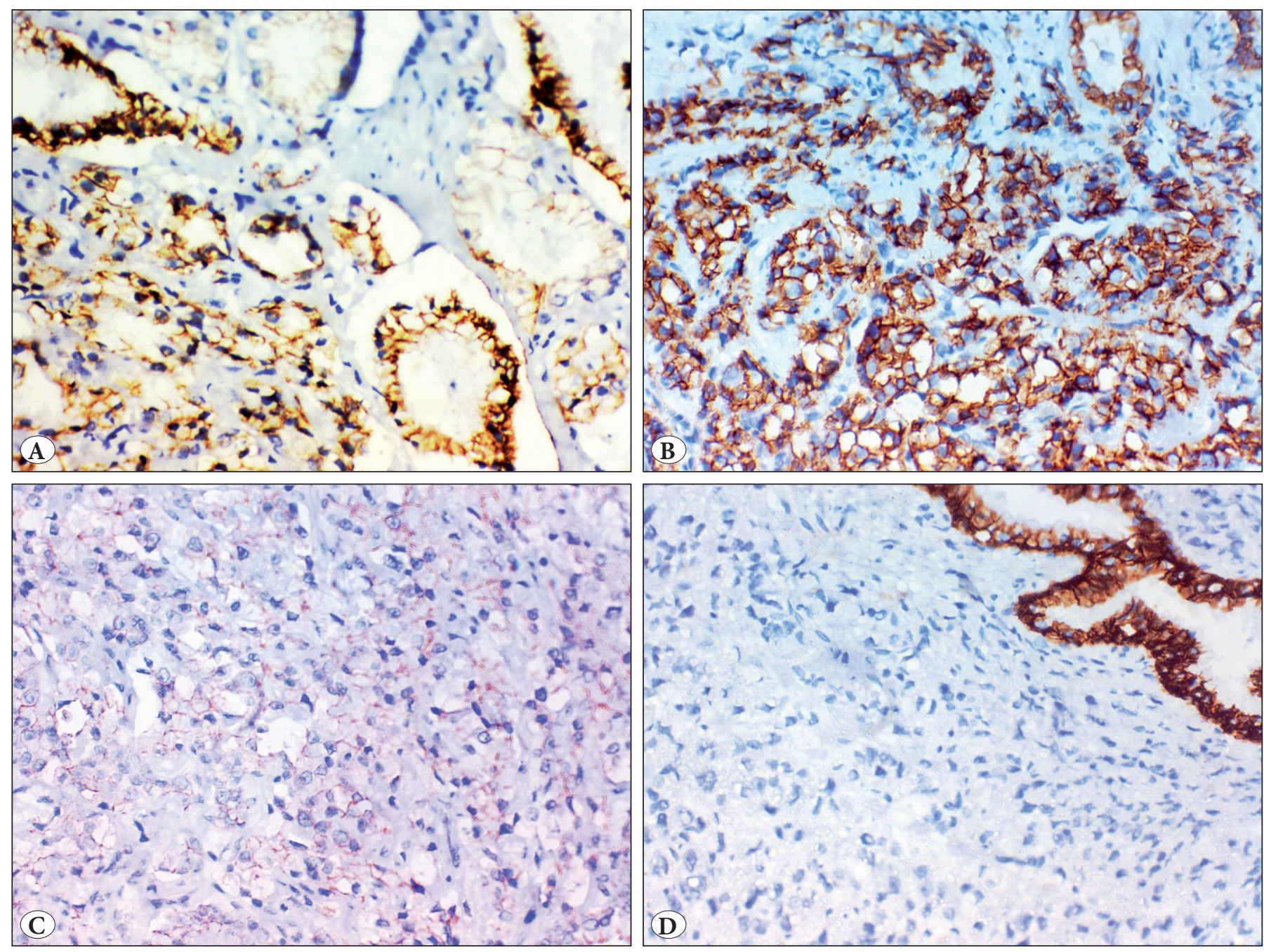

Figure 3: A) Low-grade prostate cancer (Gleason score <7) shows preserved immunoreactivity of E-cadherin (IHC; x400). B) High-grade prostate cancer (Gleason score $\geq 7$ ) shows preserved immunoreactivity of E-cadherin (IHC; x400). C) High-grade prostate cancer (Gleason score $\geq 7$ ) shows aberrant membranous immunoreactivity of E-cadherin (IHC; x400). D) High-grade prostate cancer (Gleason score $\geq 7$ ) shows completely negative immunoreactivity of E-cadherin; note the normal immunoreactivity in the benign glands (IHC; 400 ). 


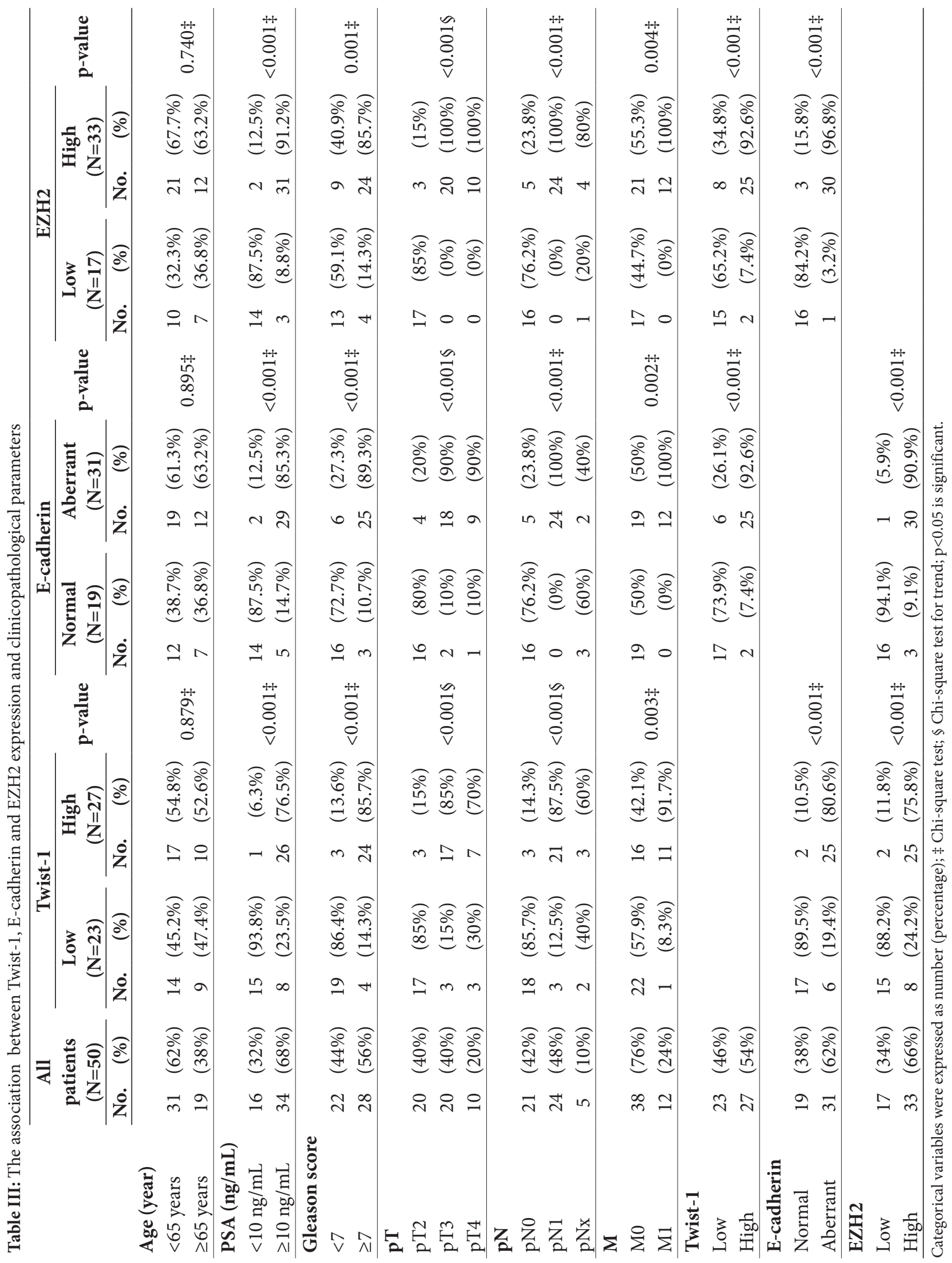


Table IV: Impact of markers on biochemical progression and biochemical progression free survival.

\begin{tabular}{|c|c|c|c|c|c|c|c|c|c|c|c|}
\hline \multirow{3}{*}{ Markers } & \multirow{3}{*}{$\begin{array}{c}\text { Total } \\
(\mathrm{N}=50)\end{array}$} & \multicolumn{4}{|c|}{ Biochemical progression } & \multirow{3}{*}{ p-value } & \multicolumn{4}{|c|}{ Biochemical Progression Free Survival } & \multirow{3}{*}{ p-value } \\
\hline & & & $\begin{array}{l}\text { bsent } \\
\mathrm{V}=35)\end{array}$ & & $\begin{array}{l}\text { resent } \\
\mathrm{N}=15)\end{array}$ & & \multirow{2}{*}{$\begin{array}{c}\text { Mean } \\
\text { (months) }\end{array}$} & \multirow[t]{2}{*}{$1 y(\%)$} & \multirow[t]{2}{*}{$2 y(\%)$} & \multirow[t]{2}{*}{$3 y(\%)$} & \\
\hline & & No. & $(\%)$ & No. & $(\%)$ & & & & & & \\
\hline All patients & 50 & 35 & $(70 \%)$ & 15 & $(30 \%)$ & & 29.4 month & $87.6 \%$ & $67.9 \%$ & $63.7 \%$ & \\
\hline \multicolumn{12}{|l|}{ Twist-1 } \\
\hline Low & 23 & 22 & $(95.7 \%)$ & 1 & $(4.3 \%)$ & $<0.001 \ddagger$ & 35.3 month & $100 \%$ & $95.2 \%$ & $95.2 \%$ & $<0.001 \dagger$ \\
\hline High & 27 & 13 & $(48.1 \%)$ & 14 & $(51.9 \%)$ & & 22.3 month & $76.7 \%$ & $39.2 \%$ & --- & \\
\hline \multicolumn{12}{|l|}{ E-cadherin } \\
\hline Normal & 19 & 16 & $(84.2 \%)$ & 3 & $(15.8 \%)$ & $0.086 \ddagger$ & 33.8 month & $100 \%$ & $89.5 \%$ & $82 \%$ & $0.013 \dagger$ \\
\hline Aberrant & 31 & 19 & $(61.3 \%)$ & 12 & $(38.7 \%)$ & & 25.7 month & $79.4 \%$ & $49.5 \%$ & --- & \\
\hline \multicolumn{12}{|l|}{ EZH2 } \\
\hline Low & 17 & 16 & $(94.1 \%)$ & 1 & $(5.9 \%)$ & $0.008 \ddagger$ & 35.5 month & $100 \%$ & $100 \%$ & $90.9 \%$ & $0.002 \dagger$ \\
\hline High & 33 & 19 & $(57.6 \%)$ & 14 & $(42.4 \%)$ & & 25.4 month & $81.4 \%$ & $47.4 \%$ & --- & \\
\hline
\end{tabular}

Categorical variables were expressed as number (percentage); $\ddagger$ Chi-square test; $§$ Chi-square test for trend; $\uparrow$ Log rank test; $\mathrm{p}<0.05$ is significant.
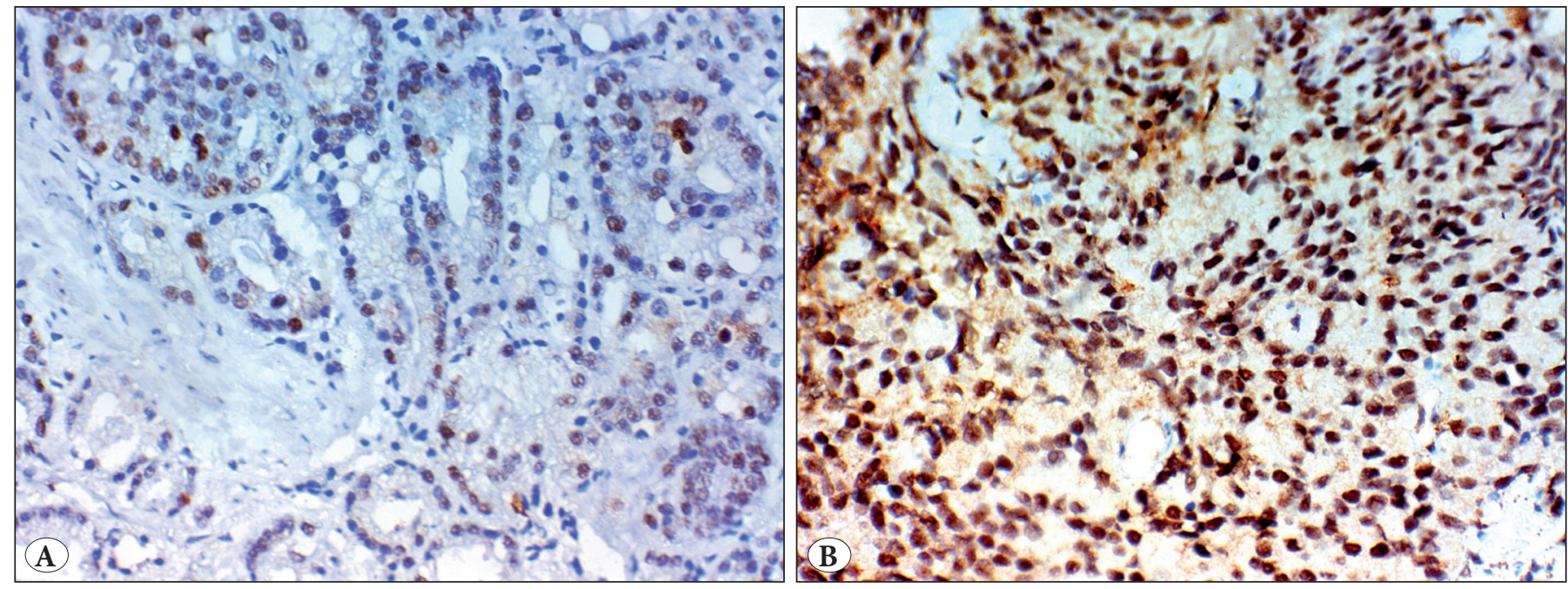

Figure 4: A) Low-grade prostate cancer (Gleason score <7) shows low nuclear immunoreactivity of EZH2 (IHC; x400). B) High-grade prostate cancer (Gleason score $\geq 7$ ) shows high nuclear immunoreactivity of EZH2 (IHC; x400).

Loss of intercellular adhesion molecules has been accepted as an initial step of malignant transformation preceding lymphovascular invasion (LVI) and distant metastasis. The key role of E-cadherin in carcinogenesis has been established, particularly in the prostate (30).

In the current study, all BPH showed preserved E-cadherin immunoreactivity while $62 \%$ PC cases designated aberrant E-cadherin immunoreactivity with a significant difference between the two groups. The membranous immunoreactivity of E-cadherin was negatively correlated with the initial PSA level and increased Gleason score. These results suggest that abnormal localization of
E-cadherin may occur during PC progression leading to loss of epithelial differentiation through the loss of cell polarity and adhesion. Furthermore, aberrant E-cadherin was significantly associated with advanced tumor stage, LN metastasis, and distant metastasis; therefore with poor prognosis. These results are hand in hand with Whiteland et al. (25). Meng et al. also reported that PC metastasis was inhibited in mice that received a high dose of zileuton, 5-lipoxygenase inhibitor, which restored normal expression of E-cadherin (31).

On the other hand, Ipekci et al.'s analysis failed to demonstrate an association between E-cadherin expression and tumor 

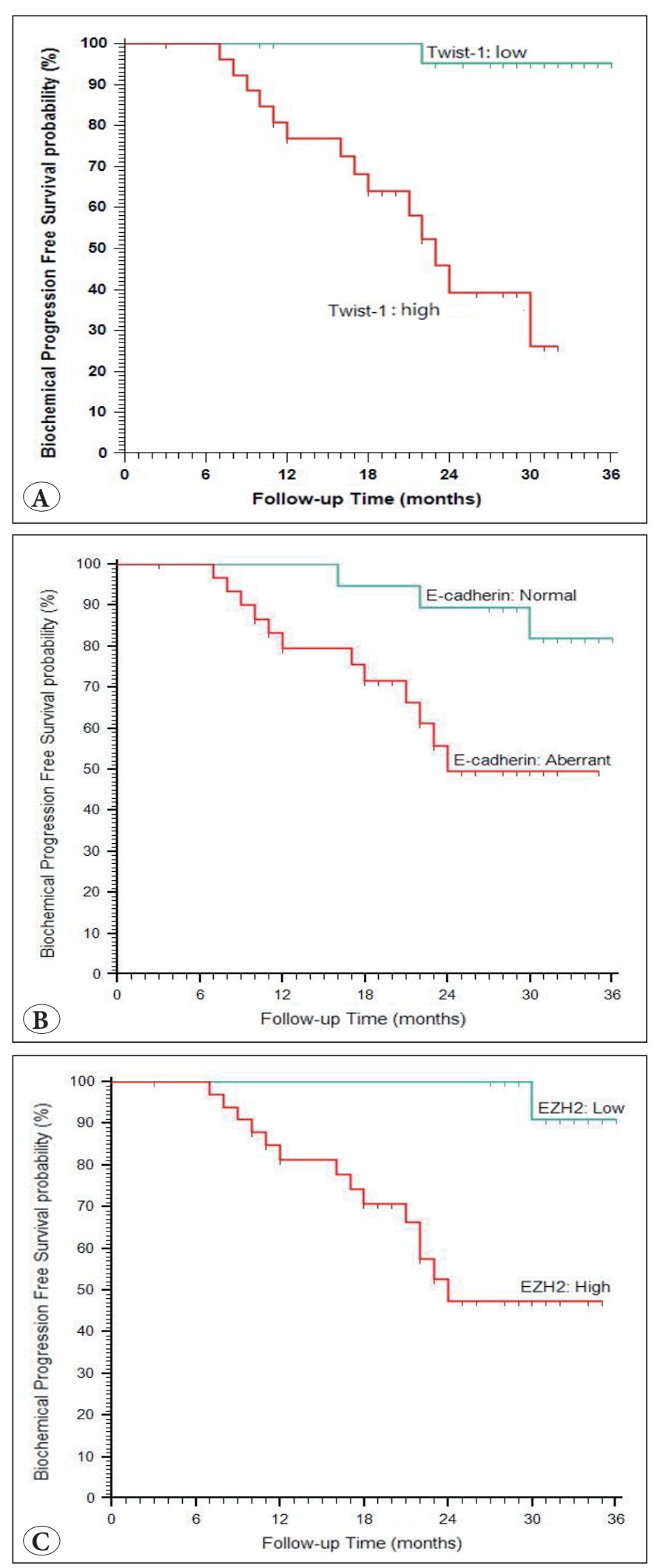

Figure 5: Kaplan-Meier plots of biochemical progression free survival (BPFS); A) Stratified according to Twist-1 expression $(\mathrm{p}<0.001)$. B) Stratified according to E-cadherin expression $(p=0.013) . C)$ Stratified according to EZH2 $(p=0.002)$. stage, grade, and initial PSA level. They explained their results with the fact that EMT is an early event in tumor progression and assessment of primary tumor does not give any insight into the metastatic potential of cells that require having other alterations to survive in distant tissues (3).

Induction of EMT by different stimuli could generate stem-like cells with enhanced self-renewal and invasive capacity with a high drug resistance, which is strongly associated with metastasis and recurrence. However, the molecular mechanisms responsible for these processes are not completely understood (7).

EZH2 increases the proliferation and invasiveness of PC cells (32). The expression of EZH2 promotes the histone deacetylase (HDAC) activity and the enhanced HDAC activity leads to removal of the acetyl group from the histone $\mathrm{H} 3 \mathrm{~K} 27$ at the E-cadherin promoter region. This helps the histone methyl-transferase activity of EZH2. Trimethylation of histone $\mathrm{H} 3$ on lysine 27 leads to chromatin compaction and transcription factors suppression from binding and initiating transcription (33). Moreover, histone deacetylase inhibitors can prevent EZH2mediated suppression of E-cadherin and attenuation of cell invasion, suggesting a possible mechanism of therapeutic treatments. In addition, a large body of evidence suggests that pharmacological inhibition of the enzymatic activity of the methyl-transferase EZH2 is an attractive therapeutic strategy for castration-resistant prostate cancer (34).

In the present study, all BPH showed low EZH2 expression while 66\% PC cases showed high EZH2 expression with a significant difference between the two groups; these results are similar to the observation of Matsika et al. (21). Moreover, a significant association was found between EZH2 with an initial PSA level, Gleason score, and TNM staging of the prostate, which confirm its crucial role in the promotion of carcinogenesis and progression as previously reported by Bryant et al. who found that EZH2 increases the proliferation and invasiveness of PC cells (32). A significant association between EZH2 and the different pathological parameters had also been reported by some investigators (21). This result contrasts with those of Varambally et al. who did not find a significant correlation between EZH2 level and these pathologic parameters (35). Laitinen et al. also reported a significant association between EZH2 and Gleason score and the initial PSA level, whereas a nonsignificant association with tumor stage $(\mathrm{pT})$ was found (36).

In our study, we found a significant inverse association between EZH2 and E-cadherin expression where 90.9\% 
of cases with high EZH2 expression designated aberrant E-cadherin expression whereas $94.1 \%$ of cases with low EZH2 expression showed normal E-cadherin staining. These findings confirm the suggestion of EZH2- mediated repression of E-cadherin and explain the positive correlation of EZH2 with advanced tumor stage through the loss of cellular adhesion and the enhancement of cancer cell motility. In addition, a significant association between EZH2 and Twist-1 expression was noted in our study confirming the observation of some investigator regarding the ability of EMT (upregulation of Twist-1) to generate stem like-cells (EZH2) enhancing the metastasis and recurrence of the involved tumor (7).

Regarding the association between the prognostic parameters and biochemical progression in our study, we found a significant association between the conventional parameters including the initial PSA level, Gleason score $\geq 7$, and tumor TNM stage with biochemical progression as previously reported by other investigators (37-39). In addition, early biochemical recurrence was more prevalent in cases with high Twist-1, aberrant E-cadherin, and high EZH2 expression.

In the present study, Kaplan-Meier survival curve analysis revealed a significant association between the initial PSA level, Gleason score, and TNM stage of the studied cases with BPFS as previously reported (22). In addition, a significant association was found between high Twist- 1 expression and shorter BPFS. Similarly, other investigators have reported that twist-1 expression confers a worse prognosis (22). Moreover, aberrant E-cadherin expression of PC cases revealed shorter BPFS and therefore poor prognosis. These results are hand in hand with other investigators $(22,25)$. Whiteland et al. demonstrated that reduced E-Cadherin expression was linked to worse prognosis and poor diseasefree survival, where E-cadherin was the only EMT marker significantly associated with patients who died as a result of PC progression when its expression was lost (25). On the other hand, Ipekci et al. failed to find an association between aberrant E-Cadherin expression and BPFS (3).

As regards EZH2, cases with high expression revealed shorter BPFS and so poor prognosis $(p=0.002)$. This finding agreed with van Leenders et al. who confirmed that EZH2 expression in neoplastic cells had a high predictive value for cancer outcome and had an increased risk of biochemical progression (40). On the other hand, some have found that EZH2 is not an independent prognosticator and when combined with decreased expression of E-cadherin revealed a significant association with short progression-free survival (41). These studies have used tissue microarrays
(TMAs) and core biopsies which, by their nature, limit tumor sampling and do not take into account the tumor heterogeneity and patchy staining expected of stem cell markers. The differences between our results and this study may also be due to using different antibody clones. Therefore, these observations support the suggestion that EZH2 can serve as a beneficial biomarker for prognostic purposes in a $\mathrm{PC}$, as respect to risk evaluation and prognosis prediction. EZH2 has also been suggested as a target for PC immunotherapy as EZH2-based peptides are detected by cytotoxic T cells and immunoglobulin G in PC patients (42). Therefore, understanding the complex steps of EMT and metastasis will help in the development of improved anti-metastatic drug strategies that are helpful against the circulating metastatic cells and therapy-resistant cancer cells (43).

In conclusion, the high Twist-1 expression, aberrant E-cadherin expression and high EZH2 in primary PC are considered as adverse prognostic markers of an aggressive tumor with high metastatic potential. Assessment of their expression level would help in the accurate prediction of the biochemical progression. Therefore, a standardized clinical trial with a larger sample is required to assess the value of these biomarkers as targeted therapy.

\section{CONFLICT of INTEREST}

The authors declare that they have no conflict of interest.

\section{REFERENCES}

1. Siegel RL, Miller KD, Jemal A. Cancer statistics, 2016. CA Cancer J Clin. 2016;66:7-30.

2. NCCN. Clinical practice guidelines in oncology: Prostate cancer. National comprehensive cancer network 2014; Version 1: Available from: NCCN.org. Detailed guidelines on the detection, prevention, risk stratification and treatment of patients.

3. Ipekci T, Ozden F, Unal B, Saygin C, Uzunaslan D, Ates E. Epithelial-mesenchymal transition markers $\beta$-catenin, snail, and e-cadherin do not predict disease free survival in prostate adenocarcinoma: A prospective study. Oncol Res. 2015;21:120916.

4. Li P, Yang R, Gao W. Contributions of epithelial-mesenchymal transition and cancer stem cells to the development of castration resistance of prostate cancer. Mol Cancer. 2014;13:55.

5. Celià-Terrassa T, Meca-Cortés $\mathrm{O}$, Mateo F, de Paz AM, Rubio N, Arnal-Estapé A, Ell BJ, Bermudo R, Díaz A, Guerra-Rebollo M, Lozano JJ, Estarás C, Ulloa C, Álvarez-Simón D, Milà J, Vilella R, Paciucci R, Martínez-Balbás M, de Herreros AG, Gomis RR, Kang Y, Blanco J, Fernández PL, Thomson TM. Epithelial mesenchymal transition can suppress major attributes of human epithelial tumor-initiating cells. J Clin Invest. 2012;122:1849-68. 
6. Sun Y, Wang BE, Leong KG, Yue P, Li L, Jhunjhunwala S, Chen D, Seo K, Modrusan Z, Gao WQ, Settleman J, Johnson L. Androgen deprivation causes epithelial-mesenchymal transition in the prostate: Implications for androgen-deprivation therapy. Cancer Res. 2012;72:527-36.

7. Kong D, Li Y, Wang Z, Sarkar FH. Cancer stem cells and epithelialto-mesenchymal transition (EMT)-phenotypic cells: Are they cousins or twins? Cancers. 2011;3:716-29.

8. Lemma S, Karihtala P, Haapasaari KM, Jantunen E, Soini Y, Bloigu R, Pasanen AK, Turpeenniemi-Hujanen T, Kuittinen O. Biological roles and prognostic values of the epithelialmesenchymal transition-mediating transcription factors Twist, ZEB1and Slug in diffuse large B-cell lymphoma. Histopathology. 2013;62:326-33.

9. Lamouille $S, X u$ J, Derynck R. Molecular mechanisms of epithelial mesenchymal transition. Nat Rev Mol Cell Biol. 2014;15:178-96.

10. Qin Q, Xu Y, He T, Qin C, Xu J. Normal and disease-related biological functions of Twist1 and underlying molecular mechanisms. Cell Res. 2012;22:90-106.

11. Gajula RP, Chettiar ST, Williams RD, Thiyagarajan S, Kato Y, Aziz K, Wang R, Gandhi N, Wild AT, Vesuna F, Ma J, Salih T, Cades J, Fertig E, Biswal S, Burns TF, Chung CH, Rudin CM, Herman JM, Hales RK, Raman V, An SS, Tran PTet. The twist box domain is required for Twist1-induced prostate cancer metastasis. Mol Cancer Res. 2013;11:1387-400.

12. Chunthapong J, Seftor EA, Khalkhali-Ellis Z. Seftor REB, Amir S, Lubaroff DM, Heidger PM, Hendrix MJC. Dual roles of E-cadherin in prostate cancer invasion. J Cell Biochem. 2004;91;649-61.

13. David J, Rajasekaran A. Dishonorable discharge: The oncogenic roles of cleaved E-cadherin fragments. Cancer Res. 2012;72:291723.

14. Chou RH, Yu YL, Hung MC. The roles of EZH2 in cell lineage commitment. Am J Transl Res. 2011;3:243-50.

15. Yang YA, Yu J. EZH2, an epigenetic driver of prostate cancer. Protein Cell. 2013;4:331-41.

16. Nolan KD, Franco OE, Hance MW, Hayward SW, Isaacs JS. Tumor-secreted Hsp90 subverts polycomb function to drive prostate tumor growth and invasion. J Biol Chem. 2015;290:827182.

17. Epstein JI1, Allsbrook WC Jr, Amin MB, Egevad LL; ISUP Grading Committee. The 2005 International Society of Urological Pathology (ISUP) consensus conference on Gleason grading of prostatic carcinoma. Am J Surg Pathol. 2005;29:1228-42.

18. Edge SB and Compton CC. The American Joint Committee on Cancer: The 7th edition of the AJCC cancer staging manual and the future of TNM. Ann Surg Oncol. 2010;17:1471-4.

19. Lei P, Ding D, Xie J, Wang L, Liao Q, Hu Y. Expression profile of Twist, vascular endothelial growth factor and CD34 in patients with different phases of osteosarcoma. Oncol Lett. 2015;10:41721.

20. Yuen HF, Chua CW, Chan YP, Wong YC, Wang X, Chan KW. Significance of TWIST and E-cadherin expression in the metastatic progression of prostatic cancer. Histopathology. 2007;50:648-658.
21. Matsika A, Srinivasan B, Day C, Mader SA, Kiernan DM, Broomfield A, Fu J, Hooper JD, Kench JG, Samaratunga H. Cancer stem cell markers in prostate cancer: An immunohistochemical study of ALDH1, SOX2 and EZH2. Pathology. 2015;47:622-8.

22. Behnsawy HM, Miyake H, Harada K, Fujisawa M. Expression patterns of epithelial-mesenchymal transition markers in localized prostate cancer: significance in clinicopathological outcomes following radical prostatectomy. BJU Int. 2013; 111:307.

23. Hoogland AM, Kweldam CF, van Leenders LH. Prognostic histopathological and molecular markers on prostate cancer needle-biopsies: A review. Biomed Res Int. 2014;2014:341324.

24. Shiota M, Izumi H, Onitsuka T, Miyamoto N, Kashiwagi E, Kidani A, Yokomizo A, Naito S, Kohno K. Twist promotes tumor cell growth through YB-1 expression. Cancer Res. 2008;68:98105.

25. Whiteland H, Spencer-Harty S, Thomas DH, Davies C, Morgan C, Kynaston H, Bose P, Fenn N, Lewis PD, Bodger O, Jenkins S, Doak SH. Putative prognostic epithelial-to-mesenchymal transition biomarkers for aggressive prostate cancer. Exp Mol Pathol. 2013;95:220-6.

26. Lee TK, Poon RT, Yuen AP Ling MT, Kwok WK, Wang XH, Wong YC, Guan XY, Man K, Chau KL, Fan ST. Twist overexpression correlates with hepatocellular carcinoma metastasis through induction of epithelial-mesenchymal transition. Clin Cancer Res. 2006;12:5369-76.

27. Kwok WK, Ling MT, Lee TW, Lau TC, Zhou C, Zhang X, Chua CW, Chan KW, Chan FL, Glackin C, Wong YC, Wang X: Upregulation of TWIST in prostate cancer and its implication as a therapeutic target. Cancer Res. 2005; 65:5153-62.

28. Buehler D, Hardin H, Shan W, Montemayor-Garcia C, Rush PS, Asioli S, Chen H, Lloyd RV. Expression of epithelial-mesenchymal transition regulators SNAI2 and TWIST1 in thyroid carcinomas. Mod Pathol. 2013; 26:54-61.

29. Zeng J, Zhan P, Wu G, Yang W, Liang W, Lv T, Song Y. Prognostic value of twist in lung cancer: Systematic review and meta-analysis. Transl Lung Cancer Res. 2015;4:236-41.

30. Lazari P, Poulias H, Gakiopoulou H, Thomopoulou GH, Barbatis C, Lazaris AC. Differential immunohistochemical expression of CD44s, E-cadherin and $\beta$-catenin among hyperplastic and neoplastic lesions of the prostate gland. Urol Int. 2013;90:109-16.

31. Meng Z, Cao R, Yang Z, Liu T, Wang Y, Wang X. Inhibitor of 5-lipoxygenase, zileuton, suppresses prostate cancer metastasis by upregulating E-cadherin and paxillin. Urology. 2013;82:1452.e714.

32. Bryant RJ, Cross NA, Eaton CL, Hamdy FC, Cunliffe VT. EZH2 promotes proliferation and invasiveness of prostate cancer cells. Prostate. 2007;67:547-56.

33. Cao Q, Yu J, Dhanasekaran SM, Kim JH, Mani RS, Tomlins SA, Mehra R, Laxman B, Cao X, Yu J, Kleer CG, Varambally S, Chinnaiyan AM. Repression of E-cadherin by the polycomb group protein EZH2 in cancer. Oncogene. 2008;27:7274-84. 
34. Xu K, Wu ZJ, Groner AC, He HH, Cai C, Lis RT, Wu X, Stack EC, Loda M, Liu T, Xu H, Cato L, Thornton JE, Gregory RI, Morrissey C, Vessella RL,Montironi R, Magi-Galluzzi C, Kantoff PW, Balk SP, Liu XS, Brown M. EZH2 oncogenic activity in castrationresistant prostate cancer cells is Polycomb-independent. Science. 2012;338:1465-9.

35. Varambally S, Dhanasekaran SM, Zhou M, Barrette TR, KumarSinha C, Sanda MG, Ghosh D, Pienta KJ, Sewalt RG, Otte AP, Rubin MA, Chinnaiyan AM. The polycomb group protein $\mathrm{EZH} 2$ is involved in progression of prostate cancer. Nature. 2002;419:624-9.

36. Laitinen S, Martikainen PM, Tolonen T, Isola J, Tammela TL, Visakorpi T. EZH2, Ki-67 and MCM7 are prognostic markers in prostatectomy treated patients. Int J Cancer. 2008;122:595-602.

37. Uemura H, K. Hoshino, T. Sasaki Miyoshi Y, Ishiguro H, Inayama Y, Kubota Y. Usefulness of the 2005 International Society of Urologic Pathology Gleason grading system in prostate biopsy and radical prostatectomy specimens. BJU Int. 2009;103:1190-4.

38. Nonomura N, Takayama H, Nakayama M, Nakai Y, Kawashima A, Mukai M, Nagahara A, Aozasa K, Tsujimura A. Infiltration of tumour-associated macrophages in prostate biopsy specimens is predictive of disease progression after hormonal therapy for prostate cancer. BJU Int. 2011;107:1918-22.
39. Pierorazio PM, Ross AE, Lin BM, Epstein JI, Han M, Walsh PC, Partin AW, Pavlovich CP, Schaeffer EM. Preoperative characteristics of high-Gleason disease predictive of favourable pathological and clinical outcomes at radical prostatectomy. BJU Int. 2012;110:1122-8.

40. van Leenders GJ1, Dukers D, Hessels D, van den Kieboom SW, Hulsbergen CA, Witjes JA, Otte AP, Meijer CJ, Raaphorst FM. Polycomb-group oncogenes EZH2, BMI1, and RING1 are overexpressed in prostate cancer with adverse pathologic and clinical features. Eur Urol. 2007;52:455-63.

41. Rhodes DR, Sanda MG, Otte AP, Chinnaiyan AM, Rubin MA. Multiplex biomarker approach for determining risk of prostatespecific antigen-defined recurrence of prostate cancer. J Natl Cancer Inst. 2003;95:661-8.

42. Ogata R, Matsueda S, Yao A, NoguchiM, Itoh K, Harada M. Identification of polycomb group protein enhancer of zeste homolog 2 (EZH2)-derived peptides immunogenic in HLA-A24+ prostate cancer patients. Prostate. 2004;60:273-81.

43. Heerboth S, Housman G, Leary M, Longacre M, Byler S, Lapinska K, Willbanks A, Sarkar S. EMT and tumor metastasis. Clin Transl Med. 2015;4:6. 